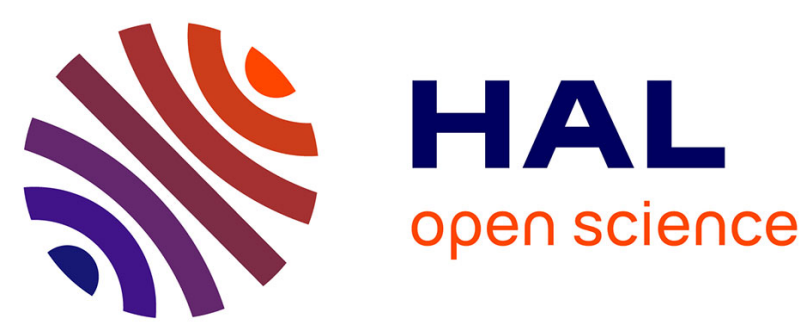

\title{
Thermal aspects of a micro thermal conductivity detector for micro gas chromatography
}

\author{
Hany A Ali, Michele Pirro, Patrick Poulichet, William Cesar, Frédéric Marty, \\ Imadeddine Azzouz, Martine Gnambodoe-Capochichi, E. Nefzaoui, Tarik \\ Bourouina
}

\section{To cite this version:}

Hany A Ali, Michele Pirro, Patrick Poulichet, William Cesar, Frédéric Marty, et al.. Thermal aspects of a micro thermal conductivity detector for micro gas chromatography. 2019 Symposium on Design, Test, Integration \& Packaging of MEMS and MOEMS (DTIP), May 2019, Paris, France. pp.1-6, 10.1109/DTIP.2019.8752795 . hal-02182952

\section{HAL Id: hal-02182952 https://hal.science/hal-02182952}

Submitted on 14 Jul 2019

HAL is a multi-disciplinary open access archive for the deposit and dissemination of scientific research documents, whether they are published or not. The documents may come from teaching and research institutions in France or abroad, or from public or private research centers.
L'archive ouverte pluridisciplinaire HAL, est destinée au dépôt et à la diffusion de documents scientifiques de niveau recherche, publiés ou non, émanant des établissements d'enseignement et de recherche français ou étrangers, des laboratoires publics ou privés. 


\title{
Thermal aspects of a micro thermal conductivity detector for micro gas chromatography
}

\author{
Hany A. Ali, Michele Pirro, Patrick Poulichet, William Cesar, Frederic Marty, Imadeddine Azzouz, Martine Gnambodoe-Capochichi, \\ $†$ Elyes Nefzaoui and Tarik Bourouina \\ Université Paris-Est, ESYCOM (FRE2028), CNAM, CNRS, ESIEE Paris, Université Paris-Est Marne-la-Vallée, \\ F-77454 Marne-la-Vallée, France \\ †Corrosponding author: elyes.nefzaoui@esiee.fr
}

\begin{abstract}
We report on a micro Thermal Conductivity Detector $(\mu \mathrm{TCD})$ design, simulation, fabrication and experimental characterization. The considered detector has been fabricated and integrated in a full micro gas chromatography device. It has been experimentally tested for different carrier gases, air, nitrogen and ethanol with different concentrations of three pollutants, Toluene, Ethylbenzene, and Xylene for instance. The device reveals to be sensitive for concentrations as low as 500 ppm. We consider different operation modes under constant temperature and constant current. We also consider steady state and transient operation modes. We show that for the same device, sensitivity strongly depends on operation mode. In addition to sensitivity enhancement, the transient operation mode enables energy consumption reduction and fast responses with measurements as fast as $0.9 \mathrm{~ms}$. Experimental results are in very good agreement with 3D numerical multi-physical simulations based on Finite Element Method.
\end{abstract}

Keywords- Thermal conductivity detector, chemical sensor, Transient, Micro-fabrication

\section{INTRODUCTION}

Air pollution and quality is one of the main challenges of the $21^{\text {st }}$ century. Indeed, anthropic activity, in addition to climate change issues, induces immediate problems such as air pollution with major consequences on human health and mortality. More than $90 \%$ of global population breathes air which quality is below the World Health Organization standards and 1 of 9 deaths is due to air pollution[1]. Air quality and pollution do not refer to ambient (outdoor) air only. Indoor air quality (IAQ) and household air pollution are also major concerns if we consider their consequences on human health[2]-[4]. Air pollution reduction is therefore a priority target for countries, cities, and global organizations. A first step towards this goal lays on proper measurement of AQ. For this goal, gas Chromatography (GC) systems can be very useful. However, several challenges have to be addressed before a massive rollout of such devices for IAQ monitoring[5]. Their miniaturization is a major one.

In the present paper, we report on the fabrication, experimental characterization and modeling of a micro thermal conductivity detector ( $\mu$ TCD) to be used as part of a micro gas chromatography $(\mu \mathrm{GC})$ device for IAQ monitoring in general, and Volatile Organic Compounds (VOCs) detection in particular[6]-[10].
The present manuscript is organized as follows: first, we present the methods used to fabricate and calibrate and experimentally characterize the device and numerically simulate its behavior. Then, we report experimental results of the $\mu$ TCD response in steady state using mono carrier gases and contaminated samples with different pollutants. Experimental steady state responses are then compared to numerical predictions. We finally show, using numerical simulations, that the device performances in terms of sensitivity and energy consumption can be improved by alternative operation modes[11], [12].

\section{METHOD}

\section{A. Fabrication}

The fabrication of the $\mu$ TCD is carried out on a double side polished 500- $\mu \mathrm{m}$ thick silicon wafer. A silicon nitride layer $\mathrm{Si}_{\mathrm{x}} \mathrm{N}_{\mathrm{x}}$ (300-nm thick) is first deposited on the substrate. This layer is patterned to serve as a base for the platinum wire ( 3 in Fig 1.b). A lift-off process is then used to make the platinum wire which is $450-\mathrm{nm}$ thick, $1-\mathrm{mm}$ long and $10-\mu \mathrm{m}$ wide (4 in Fig 1.b).

Inlet and outlet access holes (1 and 2 in Fig 1.b) are then etched into the silicon wafer by deep reactive ion etching (DRIE) using an anisotropic standard Bosch process. As a result, the fluid perpendicularly flows across the TCD detector. An additional isotropic etching step is used to obtain the suspended structure. To close the chamber, a second glass wafer $(500-\mu \mathrm{m}$ thick) is structured and anodically bonded to the silicon wafer (5 in Fig 1.b). A SEM image of the resulting structure is shown in Fig. 1.a. It shows the 3D geometry of the $\mu \mathrm{TCD}$ Pt-resistor: a zigzag structure embedded in a microchannel where the sample gas flows. The metallic resistor is suspended from both ends above a silicon substrate and encapsulated in a glass chamber. We folded the platinum wire with a $\mathrm{Si}_{\mathrm{x}} \mathrm{N}_{\mathrm{x}}$ insulating layer underneath avoiding the downward thermal losses through silicon substrate. 


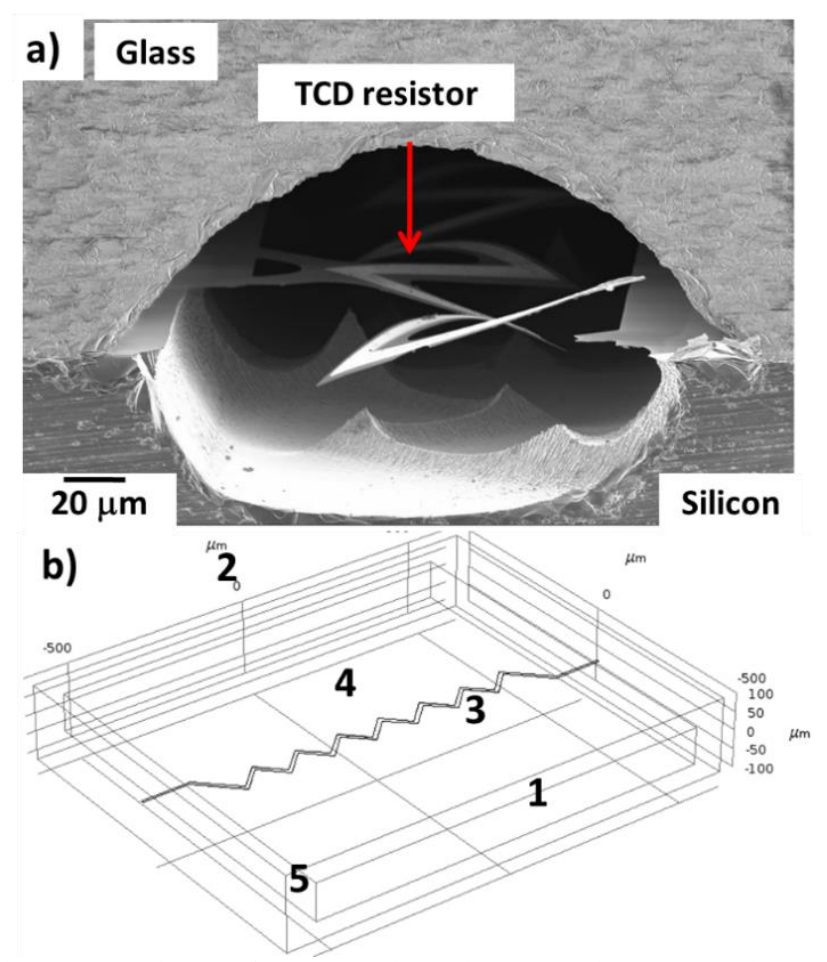

Fig. 1.a) SEM image of the suspended resistance used as a $\mu$ TCD. b) The modelled geometry used in numerical simulation under COMSOL. The simulated gas flow is perpendicular to the TCD resistor direction.

The obtained $\mu \mathrm{TCD}$ is then integrated in a full $\mu \mathrm{GC}$ device co-integrating the three main blocks of a gas chromatography system: a pre-concentrator for injection, a column for separation, and finally, a TCD for detection. More information on the full system and other sub-components can be found in [6].

\section{B. Calibration}

The behavior of the TCD is first characterized as a function of temperature. Resistance temperature coefficient and nominal resistance value are measured by placing the TCD inside an oven and by connecting the device to an ADC card with the MCP3424 (18 bits of resolution) connected to a Raspberry Pi micro-controller. For each value of the oven temperature, a differential measurement of the resistor is performed when a steady-state condition is reached. Finally, a linear relationship between the TCD resistor and temperature is determined. This relationship is used later to deduce temperature from a resistance measurement. The linear dependence of the metallic resistor resistance value on temperature is clearly observed on results plotted in Fig. 2. This figure also shows a very good agreement between measured and simulated resistance as a function of temperature.

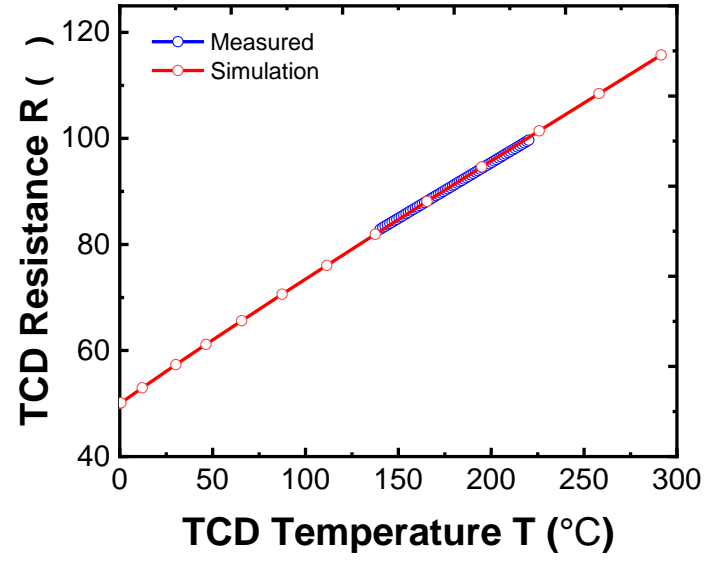

Fig. 2. The linear resistance-temperature dependence of the simulated $\mathrm{Pt}$ based-TCD with the measured one in Air as a gas carrier.

\section{Experimental setup}

All experiments are performed in a grey-room in order to control air contamination, temperature and humidity. We show a schematic of the experimental setup in Fig. 3.

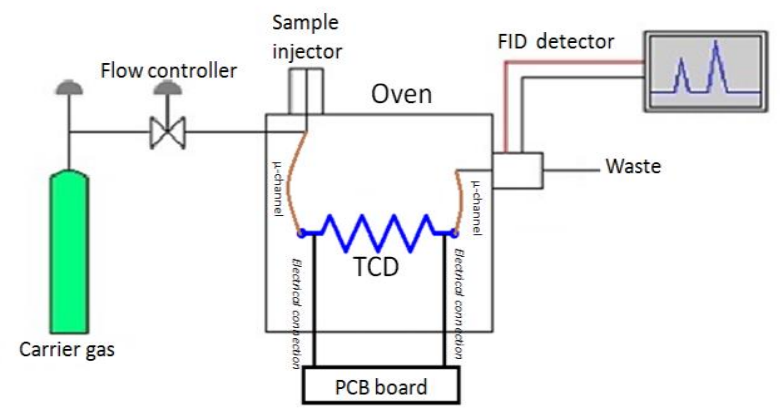

Fig. 3. Schematic of the experimental set-up

The TCD is placed inside the oven of a commercial Gas Chromatography (GC) system (Perkin Elmer Clarus 680) in order to precisely $\left(0.05^{\circ} \mathrm{C}\right.$ accuracy) control its ambient temperature. At the same time, the TCD is connected to a PCB board, outside the oven. As depicted in Fig. 3, the carrier gas is injected in the GC system and is mixed with the sample to characterize. The gas mixture reaches the TCD through a $\mu$ channel, a silica tube for instance, $100-\mu \mathrm{m}$ in diameter. After going through the TCD, the gas is directed to the Flame Ionization Detector (FID) of the GC system. The FID output is used to identify the nature and concentration of the gas mixture from the obtained chromatogram[13]. The gas preparation slightly changes, depending on the type of gas. When nitrogen is the gas under study, the TCD is first filled with air. Then, nitrogen is pumped directly into the MEMS inlet using a syringe. On the other hand, when VOC solutions are under study, a certain volume is injected in Tedlar ${ }^{\circledR}$ bags already filled with $2 \mathrm{~L}$ of nitrogen. The injected VOCs volume determines the concentration of the solution.

\section{Simulation}

We perform 3-dimensional multi-physical numerical simulations (electro-thermal, fluidic, and thermal to include Joule effect for heat generation mainly by conductive heat 
transfer across the $\mathrm{Pt}$ wire and the metal resistor with the adjacent fluid gas at its interface); we would like to remark that no heat transfer due to convention is recognizable in this model as we show later. Such a model aims at simulating and optimizing the device design and operating parameters. Simulations are based on the Finite Elements Method (FEM).

We show in Fig 1.b the geometry of the TCD as well as the simulation domain. Here, the TCD detector is embedded in a rectangular micro-channel. The channel is $200-\mu \mathrm{m}$ high, $1-\mathrm{mm}$ wide and $1-\mathrm{mm}$ deep. The $\mu \mathrm{TCD}$ is centered in the microchannel. Assuming such a channel to be rectangular simplifies the mesh used toward prompt numerical simulations. The size of the channel is set large enough to consider the outer frame at room temperature. Constant temperature boundary condition is then assumed.

Temperature dependent physical properties are used in the simulation process in order to reproduce the actual behavior of the device. This is of paramount importance for platinum wire which resistance strongly depends on temperature.

The TCD temperature; hence its resistance, is a function of the applied current, the fluid carrier, pollutant gas content and concentration. To define a temperature-based parameter in a mixture, we use a linear combination equation considering the concentration of each gas component. To this end, both transient and steady state simulations are performed.

\section{RESULTS AND DISCUSSION}

We show in Fig. 4 the value of the resistance as a function of the TCD supply current for three different gases: Air, Nitrogen $\left(\mathrm{N}_{2}\right)$ and Ethanol. Calculated errors on the resistance value are is of $\pm 0.5,0.9$ and $0.2 \Omega$ in Air, $\mathrm{N}_{2}$ and ethanol, respectively. Error bars are shown in the figure but too small to be noted.

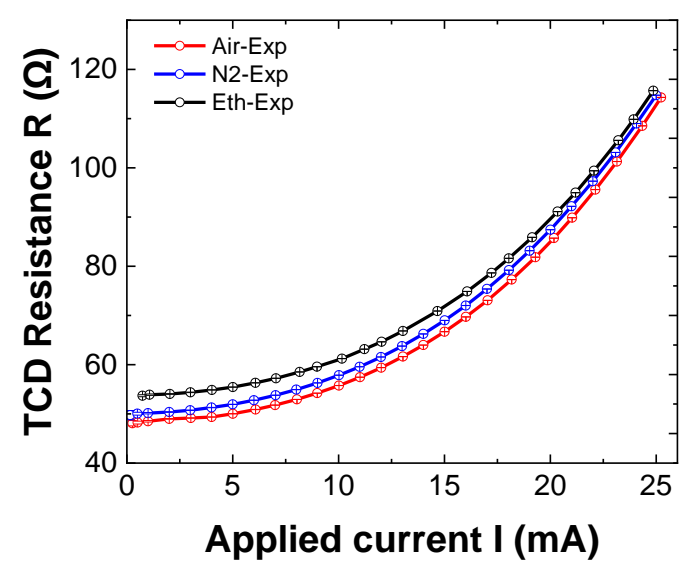

Fig. 4. Measurement of the resistance of the $\mu$ TCD under different supply currents and for different types of carrier gases.

Air and Nitrogen are common carrier gases. The TCD temperature can be calculated from the measured resistance value using a linear relationship of the resistance temperature dependence obtained during the calibration process. As shown in Fig. 4, the three different gases, due to the difference of their thermal and thermo-physical properties, can be clearly distinguished from the device response. In addition, the relative difference between the device responses to two different carrier gases depends on the operation mode: under constant resistance, i.e. constant temperature, and under constant current. Consequently, the device sensitivity depends on the operation mode. Finally, we observe a decrease of this sensitivity for large values of current and resistance, i.e. for large temperatures.

After characterization under individual carrier gases, the $\mu \mathrm{TCD}$ response is measured using a mixture of a carrier gas, nitrogen for instance, and different pollutants (Ethylbenzene, Toluene and Xylene). Measurements are performed under the following conditions: 500-ppm of pollutant concentration, 40 $\mu \mathrm{l}$ of sample volume, $30 \mathrm{psi}$ and 15 splits.

The supply current is varied and the values of the $\mu$ TCD resistance as well as the peak to peak voltage are recorded. The detector temperature is then deduced from the resistance value. Results are shown in Fig. 5 where voltage is plotted as a function of temperature for three gas samples.

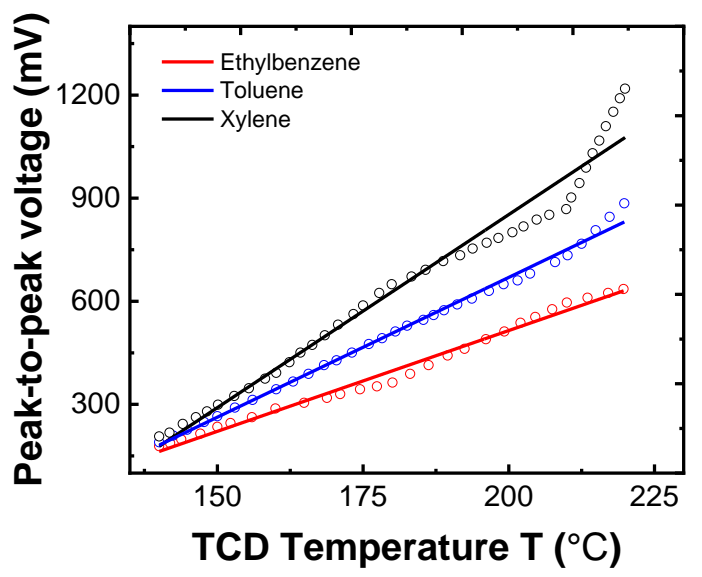

Fig. 5. Measurement of the Peak to Peak voltage at the output of the electronic circuit for Ethylbenzene, Toluene and Xylene gas as function of the temperature.

Our TCD can give different voltage signals under constant temperature at such a low pollutant concentration. We also observe that the signal increases with temperature. For temperatures lower than $200{ }^{\circ} \mathrm{C}$, the output voltage dependence to temperature is linear. Above this temperature, a strong deviation from linearity is observed, especially for Xylene sample.

Now, experimental results shown in Fig. 4 with individual gases are compared to numerical simulations. We plot the numerical steady state response (the detector resistance at thermal equilibrium under a given current) for different supplied currents and for three gases, Air, Nitrogen and Ethanol in Fig. 6.a, b and c compared with the measured TCD response.

The numerical model shows good agreement with experiment especially below applied current $=20 \mathrm{~mA}$. Above this value, the simulated results seem to be underestimated. This is probably because thermo-mechanical effects and deformation are not accounted for in our model. Such effects increase with temperature, hence with applied current value. We show in Fig. 6, on the same plot, simulated TCD responses 
under different carrier gases. We observe similar trends and an acceptable agreement with experimental responses.

After validation of the numerical model for different carrier gases, we further use it to explore the device response and sensitivity to various parameters such as the fluid velocity and applied current in both steady state and transient regimes.

We show in Fig. 7.a the device response for different gas velocities. No significant effect of this parameter is observed. This confirms that heat transfer is mainly dominated by conduction in the surrounding fluid as previously reported in literature[14], [15].

Now, the TCD transient response is probed under the same carrier gas. Obtained results in air are shown in Fig. 7.b. We observe an exponential increase of both the resistance value and temperature until reaching the equilibrium (or steady state) temperature. We also observe that the time constant, or the temperature increase rate, slightly depends on the supply current.

We further study the TCD response while introducing some pollutants with certain concentrations (e.g. $500 \mathrm{ppm}=0.05 \%$ of Ethylbenzene (EB), Toluene (TO)) in both steady state and transient regimes in Fig. 8. For steady state simulation, contaminated gases show different TCD temperatures at different applied currents (Fig. 8.a). We calculate the TCD temperature and compare it to that under a pure carrier, all things being equal. As expected, introducing pollutants; even at low concentration such as 500 ppm, increases the thermal conductivity of the whole mixture leading to lower steady TCD temperature. For instance, at $\mathrm{I}=10 \mathrm{~mA}$, the TCD equilibrium temperature in pure air is of $57.12{ }^{\circ} \mathrm{C}$, while contaminated air leads to an equilibrium temperature of $53.42{ }^{\circ} \mathrm{C}$. We also observe that the TCD becomes more sensitive at higher currents. However, the steady state response does not enable differentiating the mixtures of Ethylbenzene (EB), Toluene (TO) at this low concentration $(500 \mathrm{ppm})$. Steady state responses for these two contaminants are completely overlapped as shown in Fig. 8.a.

On the other hand, through transient simulations of the TCD response, we can distinguish different time constants for different pollutants as shown in Fig. 8.b under a supply current $\mathrm{I}=10 \mathrm{~mA}$. (Indeed, we plot as shown in Fig. 8.b the normalized TCD temperature evolution as a function of time under contaminated gases and under pure air. We note a time constant of $800 \mu$ s under pure air, while it is of 720 and $880 \mu$ s for 500 ppm of EB and TO, respectively.
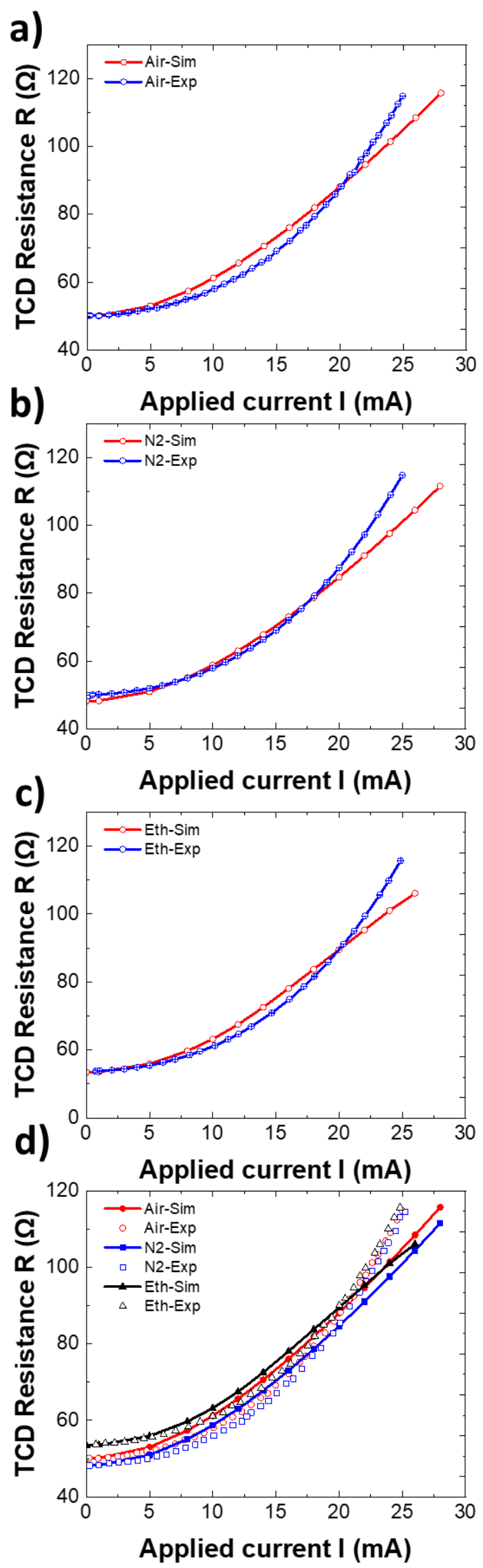
Fig. 6. Numerical steady state response of the detector as a function of current and for different pure gases, air, nitrogen and ethanol as compared with the experimental results in Figure 5. Fig 7.a, b and $\mathrm{c}$ are the TCD response for Air, $\mathrm{N}_{2}$ and Ethanol and Fig 7.d is the all together in comparison with the actual TCD measurement.
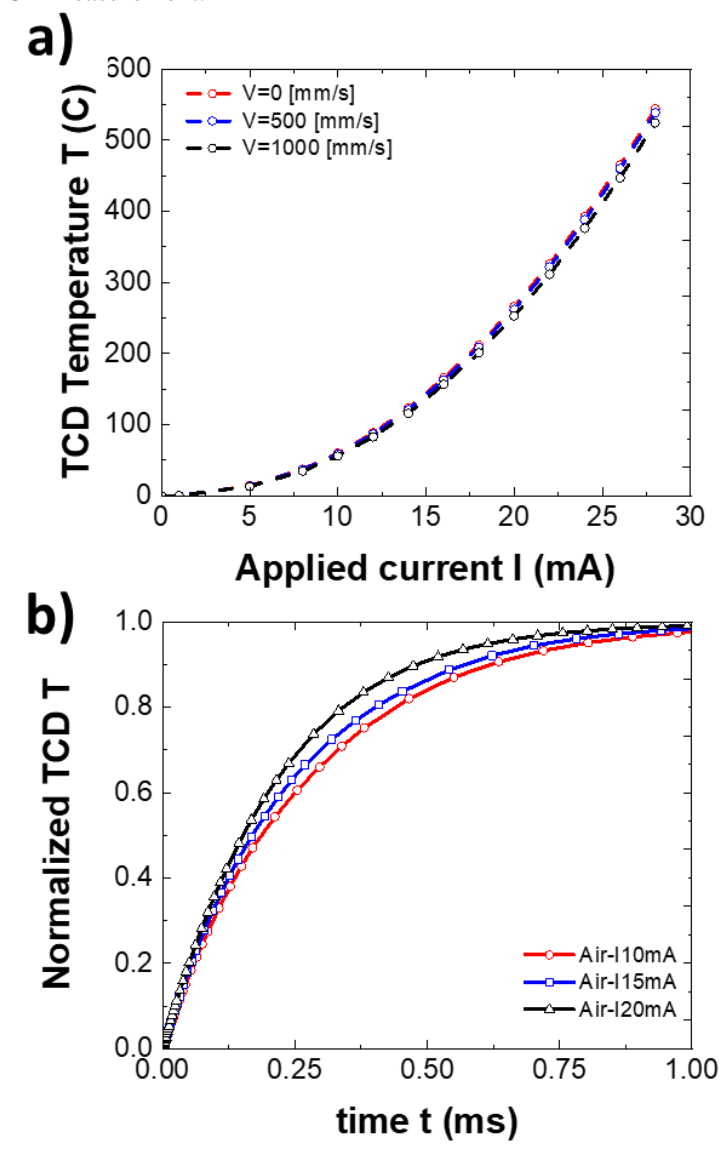

Fig. 7. Numerical simulation results under air as a gas carrier. a) is the steady state response of TCD while changing the air flow speed in the channel. b) is the transient curve of normalized temperature at different applied currents. Normalized temperature has been calculated via the equation below;

$$
D T=\frac{T-T_{0}}{T_{\max }-T_{0}} @ t=0.95=\text { time constant }
$$

These results reveal different advantages of the transient operation mode of a TCD: a better sensitivity at low concentrations, a fast response time and low energy consumption. Indeed, a measurement time lower than $900 \mu \mathrm{s}$ is required to detect such levels of contamination. Reducing measurement time, under the same supply current, significantly reduces heating time, hence the device energy consumption.
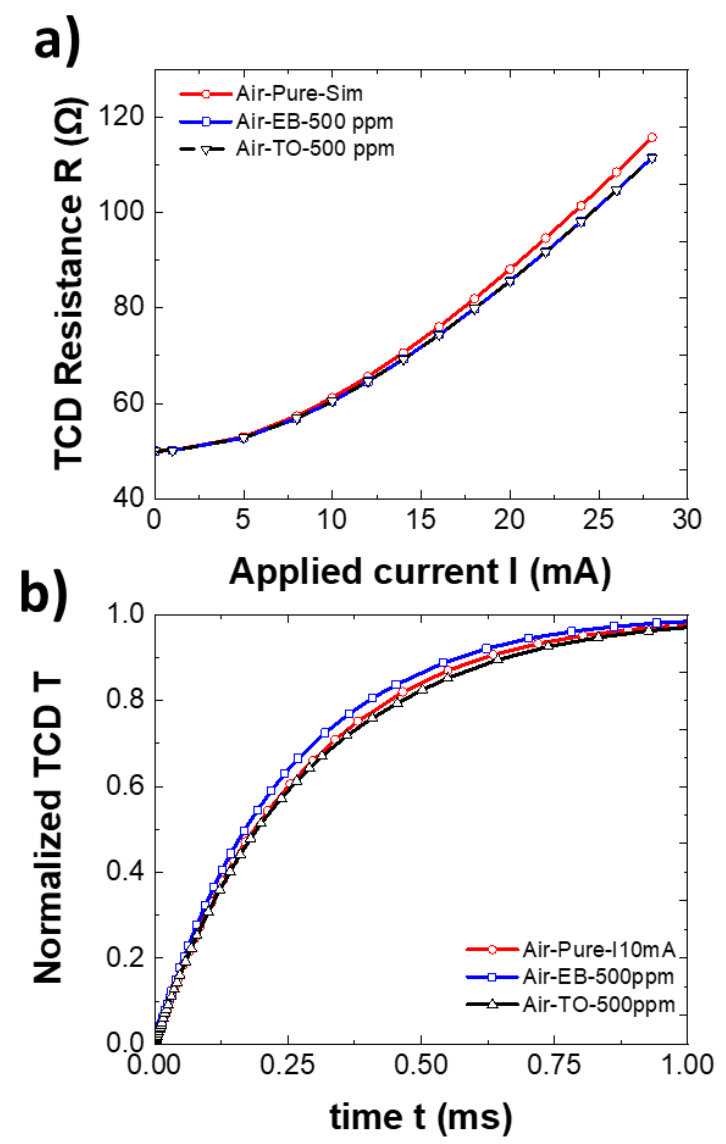

Fig. 8. Numerical results of the $\mu$ TCD in pure contaminated air with ethylbenzene and toluene with a concentration of $500 \mathrm{ppm}$. The device is assumed to be operated with a 10-mA supply current. a) is the steady state TCD response, whereas $\mathrm{b}$ ) is the TCD behavior in transient regime.

\section{CONCLUSION}

The fabrication, test and modeling of a micro thermal conductivity detector for gas sensing and VOC detection are presented in the present paper. We show, through experimental results, that the fabricated device detects pollutants concentrations as low as $500 \mathrm{ppm}$. We also note that the device sensitivity in steady state strongly depends on the operation mode, under constant current or constant temperature. A 3D multi-physical numerical model based on FEM is also described and used to understand experimental results and explore possible improvements. Numerical simulations are in very good agreement with experiment in steady state and allow us to explore transient response operation mode which reveals to be very promising. Experimental validation of transient operation performances is ongoing. Further developments are still needed to take the full benefit of this mode with respect to both energy consumption and sensitivity.

\section{ACKNOWLEDGMENT}

This work has been supported by the ANR EquipEx SENSECITY project, the FUI 18 MIMESYS funded by Region Ile-deFrance and the European Union's H2020 Program for research, technological development and demonstration under grant agreement No 644852. 


\section{REFERENCES}

[1] "State of Global Air 2018. Special Report.," Health Effects Institute, Boston, 2018.

[2] S. S. H. Ho, Y. Huang, S.-C. Lee, and J. Cao, "Challenges on field monitoring of indoor air quality in china," Indoor and Built Environment, vol. 26, no. 4, pp. 576-584, 2017.

[3] E. SCHER, "Opinion on Risk Assessment on Indoor Air Quality," European Commissions Health and Consumer Protection Directorate General, Brussel, 2007.

[4] World Health Organization and others, "Development of WHO guidelines for indoor air quality," in Report on a working group meeting, Bonn, Germany, 2006, pp. 23-24.

[5] I. Azzouz, F. Marty, and T. Bourouina, "Recent advances in microgas chromatography - The opportunities and the challenges," in 2017 Symposium on Design, Test, Integration and Packaging of MEMS/MOEMS (DTIP), 2017, pp. 1-5.

[6] I. Azzouz et al., "Evaluation of Tenax thin films as adsorbent material in a micro-preconcentrator and its operation as a valve-less multiple injection system in micro-gas chromatography," in TRANSDUCERS 2017 -

19th International Conference on Solid-State Sensors, Actuators and Microsystems, 2017, pp. 1516-1519.

[7] W. R. Collin, G. Serrano, L. K. Wright, H. Chang, N. Nuñovero, and E. T. Zellers, "Microfabricated Gas Chromatograph for Rapid, Trace-Level Determinations of Gas-Phase Explosive Marker Compounds," Anal. Chem., vol. 86, no. 1, pp. 655-663, Jan. 2014.

[8] W. R. Collin, A. Bondy, D. Paul, K. Kurabayashi, and E. T. Zellers, " $\mu \mathrm{GC} \times \mu \mathrm{GC}$ : Comprehensive Two-Dimensional Gas Chromatographic Separations with Microfabricated Components," Anal. Chem., vol. 87, no. 3, pp. 1630-1637, Feb. 2015.

[9] J. Bryant-Genevier, K. Scholten, S. K. Kim, and E. T. Zellers, "Multivariate curve resolution of co-eluting vapors from a gas chromatograph with microsensor array detector," Sensors and Actuators B: Chemical, vol. 202, pp. 167-176, Oct. 2014.

[10] L. K. Wright and E. T. Zellers, "A nanoparticle-coated chemiresistor array as a microscale gas chromatograph detector for explosive marker compounds: flow rate and temperature effects," Analyst, vol. 138, no. 22, pp. 6860-6868, Oct. 2013.

[11] A. Mahdavifar, M. Navaei, P. J. Hesketh, M. Findlay, J. R. Stetter, and G. W. Hunter, "Transient thermal response of micro-thermal conductivity detector $(\mu \mathrm{TCD})$ for the identification of gas mixtures: An ultra-fast and low power method," Microsystems \& Nanoengineering, vol. 1, p. 15025, Oct. 2015.

[12] F. Shaun et al., "Energy efficient micro-machined thermal flow-rate sensor based on transient operation mode," in 2018 Symposium on Design, Test, Integration Packaging of MEMS and MOEMS (DTIP), 2018, pp. 1-3.

[13] H. W. Lewis and C. J. Moody, "Experimental organic chemistry: Principles and practice," Nueva Jersey: WileyBlackwell, 1989.

[14] H. Berthet, J. Jundt, J. Durivault, B. Mercier, and D. Angelescu, "Time-of-flight thermal flowrate sensor for lab-on-chip applications," Lab Chip, vol. 11, no. 2, pp. 215-223, 2011.

[15] A. Mahdavifar et al., "Simulation and Fabrication of an Ultra-Low Power Miniature Microbridge Thermal Conductivity Gas Sensor," J. Electrochem. Soc., vol. 161, no. 4, pp. B55-B61, Jan. 2014. 